\title{
Royal Society of medicine.
}

\author{
Dermatologische Abteilung. \\ Sitzung rom 18. Juni 1914.
}

Davies. Zwei Fälle von Frythem infolge von Grubengasvergiftung.

Beide rührten von einer Grubengaskatastrophe im Oktober 1913 her, aus der von 500 Bergleuten nur 18 gerettet worden waren. Von diesen 18 Überlebenden, die bis zur Befreiung 20 Stunden in den Methandämpfen gewesen, zeigten 9 akutes Erythem. Dasselbe wurde auch bei einigen Leichen konstatiert, die schon nach wenigen Tagen - die Mehrzahl erst nach Wochen oder Monaten, als bereits Verwesung eingetreten - geborgen werden konnten. Die Erytheme dürften nicht Druckphänomene sein, sondern Manifestationen der Nachschwadenvergiftung.

Little, Graham demonstriert: 1. Pellagra mit Hauteruption.

17jähriger gesunder und kräftiger Junge, Engländer, erkrankte schwer im April 1913, kurz nachdem er beim Baden in einem Flusse von einer Fliege an Schulter und Skrotum gestochen worden war. Die Symptome wiesen lange Zeit auf abdominale Tuberkulose hin - z. B. Dunkel. färbung der Haut am Rücken auf Tbc. suprarenalis, ferner Diarrhoen, Pleurit exsudat. -, aber im April 1914 änderte sich das Krankheitsbild. Nach längerem Aufenthalt im Freien, in der Sonne, traten Hauterscheinungen (und gleichzeitig nervöse Störungen) auf, nämlich Rötungen und Schwellungen an den unbedeckten Körperstellen, an Stirn, Nase, Nacken und Handrücken, an diesen von den Fingern bis gegen $7 \mathrm{~cm}$ oberhalb des Gelenkes reichend mit scharfer Abgrenzung, nur auf der Streckseite. Alle diese erythematösen Stellen nahmen bald eine dunkle Färbung an, sie verschwand aber später infolge starker Abschuppungen, die eine glatte weiße Hautfläche zurückließen. Eine z weite Attacke jedoch, die Ende Mai mit 160 P. und 40-50 Respirationen eintrat, brachte die walnußbraunen Pigmentierungen an den genannten Stellen wieder hervor und außerdem noch dunklere Verfärbungen an Penis, Skrotum und Sakralgegend, während das ganze Gesicht tief gerötet und hektisch aussah. Die Pigmentierungen gingen in den nächsten Wochen zurück und starke Abschuppung setzte wieder glatte zartrote Exkoriationen an Stelle der pellagrösen Hautfarbe.

Diskussion: Die Diaguose wird bestätigt von Sequeira, der einen identischen, gleichfalls endogenen Fall behandelt, und von Pernet und Mac Leod, welche die gleichen Hautveränderungen bei vielen Pellagrösen in Italien gesehen; daß hier der Fall, der Engländer, relativ weiß ist im Vergleich zu dem Italiener, kann wohl von Klima und Nationalität herrühren. Nur Whitfield wendet ein, dab Little den Fliegenstich als Ursache der Krankheit und Stütze der Diagnose anführt, amerikanischen Autoren folgend, andererseits aber ihn im Sinne einer anderen Theorie verwendet, und daß bei Pellagra das Erythem nicht so schneil verschwinde. 


\section{Trophoedema.}

16jähriges Mädchen mit persistierendem Ödem und Adiposität der Beine von den Leisten herab bis $\mathrm{zu}$ den Knöcheln bekommt ein diffuses Erythem an den Beinen, so bald es einige Zeit auf ist, geht oder steht, während bei Ruhelage die Rötung verschwindet, Schmerzen in den Beinen aber bestehen auch dann kontinuierlich weiter. Das Leiden begann vor 3 Jahren nach einem Sturze von einem Felsen, wobei Pat. auf die FüBe fiel. Little diagnostiziert Milroys Trophödem (von anderer Seite wurde traumatische Affektion der Hypophyse, Hypopituitarismus, angenommen); es sind keine Zeichen von Myxödem vorhanden, auch nicht an den Haaren - sie sind weder überreichlich noch matt und glanzlos und es ist keine Abnormität an Thyroidea, Thymus oder anderen Irüsen wahrnehmbar.

Diskussion: Semon vermutet Myxödem, weil Haarausfall angegeben wird und die Schilddrüse nicht zu fühlen ist. Pernet nimmt Adipositas cerebralis, die mit der Zirbeldrüse in Zusammenhang steht, an; in einem Falle von dieser Krankheit hat er in Schnitten aus dem Oberschenkel keine nennenswerte Fettansammlung gefunden, nur die Haut selbst war verdickt. Die richtige Benennung wäre wohl Dermo-adipositas cerebralis.

\section{Favas bei einer Maus.}

Eine Maus mit 3 Favusherden, an Kinnbacken, Kopf und Rücken, wurde in einem von einem Ehepaar und 3 Kindern bewohnten Zimmer gefangen. Eines von diesen Kindern - die beiden anderen und die Eltern waren gesund - hatte vor einigen Monaten einen typischen Favus des Nackens mit sehr charakteristischen gelben Skutulis, aus denen das Achorion Quincke gezüchtet wurde. Schon dieser Befund wies auf eine besondere Art des Favus bei dem Kinde hin. Nun wurde jetzt aus dem Favus der Maus der gleiche Pilz in Reinkultur konstatiert.

Diskussion: Adamson hat ebenfalls, vor 6 Jahrea, Favus mit Achorion $Q u$ incke bei einem Kinde und bei einer im selben Zimmer gefangenen Maus gesehen und hält die Übertragung von diesen Mäusepilzen auf Menschen für nicht selten in England. Er selbst bat bereits 3 Fälle vorgestellt und andere wurden von $\mathrm{Mac} L \mathrm{Lod}$ und Bolam gezeigt. Es sind immer einzelne oder wenige Herde mit einem oder wenigen oder sogar ohne Skutulis, in keinem bisherigen Falle in den Haaren. Der gewöhnliche Menschenfavus scheint nur ausnabmsweise von Mäusen übertragen zu werden (Fall Pernet), bei denen außer dem Ach, Qu incke anch Ach. Sehönlein und Ach. violaceum gefunden worden sind.

Pernet. 1. Favus bei Mutter und Kind.

Die Mutter hat die Krankheit seit ihrem zweiten Lebensjahr, seit 31 Jahren, mit typischen Skutulis in den Kopf haaren; außer dem einen Kinde, bei dem Skutula aber nieht vorhanden, sollen die anderen Familienmitglieder frei von Favus sein.

Diskussion. Pringle erklärt den Favus für hänfiger in England, als man annimmt. Sequeira erinnert an den von $\mathrm{Hu}$ tchinsons und Mackenzies berühmten Fall von Favus auf der Zunge.

2. Bullöse I chthyosis.

Ein 18jähriger junger Mann, der schon im Alter von 6 Jahren 
wegen Blasen der Haut im West London Hospital behandelt worden, kam im Mai dorthin wegen Rötung und Desquamation am unteren Teile des rechten Vorderarms und mit den Resten euner bullösen Läsion an dem rechten Handrücken, die aus einer ursprünglich kleinen Blase an dem Vorder. arme dadurch entstanden ist, daß sich diese vergrößerte und auf die Hand ausdehnte. Kleinere Bullae fanden sich zerstreut auf dem linken Vorderarm und eine einzelne am linken Ellenbogen. Ferner waren erhabene rundliche, rauh ichthyotische Herde an den Ellbogen und weniger ebensolche, aber geringeren Grades, an den Vorderflächen der Knie vorhanden. Der Pat. leugnet die Bullae künstlich erzengt zu haben, und Pernet glaubt, der Fall gehöre zur Ichthyose à poussées bulleuses v. Durings oder za Brocqs Erythrodermies congénitales ichthyosiques avec hyperépiderméotrophie avec bulles.

Diskussion: Pringle möchte den Fall eher als Hystrizismus wie als Ichthyosis bezeichnen und die Bullae, die er nie dabei gesehen, als Produkte septischer Infektion. Adamson aber nimmt zoniformen Naevas an in Kombination mit Epidermolysis bullosa - gegen diese spricht allerdings das Freibleiben der Finger von Blasen - oder mit einem impetiginosen Prozesse an.

Bunch. 1. Pagets disease of the nipple.

Seit 15 Monaten an der linken Brustwarze einer 5ljäbrigen Frau; durch eine Röntgenbestrahlung so erhebliche Besserung, daß Fortsetzung dieser Behandlung richtig erscheint.

Diskussion: Die Meinungen und Erfahrungen über Röntgen. und Radiumstrablen bei dieser Krankheit gehen sehr auseinander; Sequeira, S towers und andere sahen ungünstige Resultate und halten ablatio mammae allein für richtig. Mac Gormac nimmt an, daB zwei verschiedene Karzinomarten unter dem Namen Pagetsche Krankheit passieren, eine squamöse und eine Spheroidalzellenart; die Behandlung müsse daher ebenfalls verschieden sei. Bei der letzten Art besteht Neigung zu Metastasen, nur bei der squamösen dürfe man sich expektativ verbalten und von Radium Erfolg erwarten.

2. Fall von Psoriasis zusmmen mit Thrombose der Vena cava inferior.

Koinzidenz von alter Psoriasis - typische Plaques ohne Besonderheiten - und einer vor 3 Jahren (infolge sportlicher Überanstrengungen) autgetretenen Thrombose der V. c. inf., die charakteristische Gefäßdilatationen am Abdomen herbeiführte.

Mac Leod. Lichen planus atrophicus.

Bei dem 22jährigen, sehr schwächlichen und nervösen Manne waren die Effloreszenzen in einem bräunlichen Netzwerk angeordnet, das dem Netze der oberfïachliohen Venen entsprach, an der Invenfläche des linken Knies. Es war eine handtellergrobe Plaque mit spärlichen typischen Knötchen von Lichen ruber atrophicus von braunroter Farbe, Inseln von depigmentierter Haut und daneben besonders dunkle Plaques im Netzwerk, die anscheinend ihre dunklere Färbung durch Zuwanderung des Pigments aus diesen "Inseln" bekommen hatten. Auf der Vorderfläche des rechten Kaies eine Gruppe von Lichen planus-Knötchen. Mund 
intakt. Der Fall ist interessant wegen der Schwierigkeiten der Diagnose bei flüchtiger Betrachtung, ferner aber auch wegen der Beziehungen, welche $z$ wischen Lichen planus und oberflächlichem Venennetz für die Anordnung und Verteilung der Knötchen zu bestehen scheinen.

Diskus ion: Pringle bemerkt, dab typische Effloreszenzen von Lichen spinulosus auf der korrespondierenden Partie der entgegengesetzten Seite die Diagnose des Vortragenden stützen. A dams on stimmt zu und sieht in der Verteilung der Effloreszenzen in diesem Falle eine Bestätigung seiner Ansicht, daß die Lichen planus-Knötchen dem Netzwerk der Livedo folgen.

Knowsley Sibley. Adenoma sebaceum.

Die Affektion besteht seit der Kindheit der 19jährigen, sonst stets gesunden Pat., aber in den letzten beiden Jahren sind einzelne Effloreszenzen gewachsen. Sie bestehen in erhabenen Papeln von Stecknadelkopf- bis Sagokorngröße und sind zum Teil blaß, transluzent, von wächsernem Aussehen, andere aber ausgesprochen rot und vaskulär, mit Teleangiektasien bedeckt. Das Aussehen dieser Efloreszenzen wechselt; beim Erröten der Pat, erscheinen sie mehr prominent und sollen bei der leichtesten Irritation aufbrechen und bluten. Einige von ihnen verschwinden auf Druck, andere nicht. Sie sitzen auf beiden Backen, Nasenrücken und -flügeln, Ober- und Unterlippe, sowie am Kinn; die Stirn ist frei. Leichte Seborrhoe capitis besteht, aber weder Akne und Komedonen, noch pustulöser Ausschlag im Gesicht. Ferner sind flache, nichtpigmentierte fibromatöse und einzelne kleine pigmentierte Naevi an Rumpf und Armen vorhanden, Es handelt sich um Adenoma sebaceum - auch nach dem histologischen Befunde - u. zw. um den $\mathrm{D}$ arierschen vaskulären Typus.

Sequeira. 1. Lei o m y oma.

Die 32jährige Frau hat die Affektion, so lange sie denken kann, hirse- bis erbsengroße rundliche Geschwülste auf der rechten Seite des Gesichts (an Stirn, Wange, Kinnbacke, unter dem r. Auge und an der Nase) und in einer zweiten Gruppe, nur von Hirsekorngröße, auf der linken Seite des Nackens. Allein die Tumoren auf der Stirn sind rötlich verfärbt, alle anderen von der Farbe der umgebenden Haut. Sie sollen in der kalten Jahreszeit empfindlich und schmerzhaft sein. Histologischer Befund an einem Stirntumor: Unmittelbar unter dem Epithel myomatöser Knoten aus Bündeln von glatten Muskelfasern, die von feinen kollagenen Faserbündeln durchflochten werden, ringsum eine Haarwurzel: also wohl aus den Arrektores hervorgehend.

Die unilaterale Anordnung - an Stirn und Nase bildete die Mittellinie des Gesichts genan die Grenze - und das Bestehen seit früher Kindheit ließen zunächst an Naevi denken, doch wegen der angegebenen Schmerzhaftigkeit mußten diese und mußten auch andere Hauttumoren ausgeschlossen werden.

Diskussion: Mac Leod hat gleichfalls einen streng halbseitigen Fall, von der Mitte der Wange bis zum Kinn, bei einer Frau gesehen, die ebenfalls über Schmerzen bei Kälte klagte. 
Xanthomen.

2. Díabetes insipidus mit papulösen und noduläen

Der bereits in der Dezember-Sitzung vorgestellte Fall, bei dem an Lichen planus und auch an Tuberkulide gedacht, mikroskopisch aber Granulome nachgewiesen wurde, soll ausführlich publiziert werden.

Colenso. Fall zur Diagnose.

Geschwulst am linken Ohr eines 58jährigen Mannes, die hervorgegangen sein soll aus einer angeborenen Warze (oder Naevus?). Seit einer Verletzang soll häufiges Bluten bei jedem Stol oder Schlag aufs $\mathrm{Ohr}$ eingetreten, aber erst seit $10 \mathrm{Jahren}$ allmähliche Vergrößerung erfolgt sein, bis die Geschwulst jetzt den inneren und äußeren Gehörgang: bedeckt, mit runzeliger wie zersägter Oberfläche.

Diskussion: Von mehreren Rednern wird maligne Entartung angenommen, von Mac Leod eines Naevus, von Adamson, der zwei ähnliche Fälle gesehen, und Sibley einer Talgdrüse.

Whitfield. Schambergs Krankheit.

Die Abnormität besteht in einer eigentümlichen braunroten Verfärbung der Arme von den Handgelenken bis einige Zoll oberhalb der Ellbogen und der Beine von den Malleolen bis zur Vereinigung von mittlerem und oberem Drittel der Oberschenkel. Genaue Betrachtung zeigt, daß diese braune Farbe von einem feinen Netzwerk brauner Flecke und teleangiektatischer Punkte herrührt, die auf Druck nicht verschwinden. Über dem linken Knie bestehen Teleangiektasien in ovaler Form; die Gefäße verzweigen sich nicht entlang der Zirkumferenz und hinterlassen ein gleichmäßig braun pigmentiertes Zentrum. Diese Pigmentierung bestand bei dem 11jäbrigen Knaben "schon ziemlich lange". Daher war Purpura, die nicht lange anbält, auszuschließen und auch Lichen planus wegen der GefäBerweiterung einer-, wegen des Fehlens von Knötchen andererseits. Whitfield diagnostiziort deshalb Angioma serpiginosum und $\mathrm{Schambergs}$ Disease, die er für Varietäten des gleichen Prozesses hält.

Sitzung vom 16. Juli 1914.

Little, Grabam. 1. Fall von multiplen symmetrischen schmerzhaften Fettgeschwälsten mit Pigmentation.

Bei einer Frau mittleren Alters sind in den letzten 2 Jahren zugleich mit allgemeiner Korpulenz 39 Schmerzen bereitende Geschwülste aufgetreten, davon am rechten Arm 14, am linken 13, die übrigen unregelmäßig verteilt. In letzter Zeit haben sich zu beiden Seiten des Nackens melanodermatische Plaques entwickelt. Kein Diabetes, kein pathologischer Befund im Augenhintergrund oder im Röntgenbilde der Sella turcica. stätigt.

In der Diskussion wird die Diagnose Adipositas dolorosa be-

2. Fall von akutem Lupus erythematosus.

Die Affektion begann vor 5 Monaten an der Nasenspitze, als der Pat. in Südafrika lebte, und wurde dort als Lupus erythematosus mit 
5-7 Röntgenbestrahlungen des Gesichts, sowie an den später erkrankten Händen mit einigen Sitzungen von Ätherschnee-Gefrierung behandelt. Auf der Heimreise trotz Vorsicht vor der Sonnenbestrahlung auf See erhebliche Verschlechterung. Das ganze Gesicht war, als Little ihn bald nach der Ankunft sah, stark geschwollen, fast überall intensiv gerötet, aber in diese helle Rötung ragte an beiden Jochbögen, der Stirn und dem Nacken eine dunkelbräunliche Verfärbung - ähnlich der bei Pityr. versicolor - herein, und hinter den Ohren sah man beiderseits einen bläulichroten Fleck, der infiltriert und leicht erhaben blieb, anch als die Gesichtsrötung zurückgegangen war. Die mit Ätherschnee intensiv behandelten Handrücken zeigten Flecken von weißlicher Atrophie mit einigen eingesprengten Rötungen; letztere dürften die Reste von typischem L. e., die Atrophien aber Folge intensivster Gefrierung sein.

Diskussion: Pringle schlieBt sich Littles Diagnose an, Pernet bezweifelt, daß die Pigmentierung sowie weiße Stellen an den Augenbrauen von $X$-Strahlen herrühren und glaubt eher an MelanoLeukoderma infolge der südafrikanischen Sonne; auch Adamson ver. mutet aus den gegenwärtig vorhandenen weißen Stellen im Gesicht ein primäres Leukoderm, dem Sonnenbräunung der pigmentlosen Plaques die geschilderte Bronzetönung gegeben habe, und aus der fühlbaren Verdickung der Haut an den Fingern eine Sklerodermie der Hände; Lenkoderm und Sklerodermie treten oft zusammen auf.

3. Fall von Schambergscher Kraukbeit: Eine progressive Pigmentkrankheit der Haut.

Die Affektion des 56jähr. Mannes besteht nur an den Beinen; auf dem linken begann sie vor $25-30 \mathrm{Jabren}$, auf dem rechten (wo sie jetzt größer, 5:3 ist) vor 8-10 Jahren und besteht in breiten Plaques von dichtgedrängten Pigmentationen, die wie schwarz Pfefferkörner aussehen, an den Vorderflächen der Knie; in der Umgebung dieser Plaques, die sonderbare winkelige Figuren bilden mit scharfen Linien und stellenweise serpiginösen Rändern, sind erst in den letzten Monaten einzelne solche Pigmentpunkte aufgetreten.

Diskussion: Heath, D. hat einen analogen Fall gesehen, bei dem trianguläre Pigmentplaques nicht nur an den Knien - wie Psoriasisplaques - sondern weiter verbreitet waren. Pringle hat schon eine ganze Anzahl von diesen Fällen gesehen, stets als Nebenbefund, und hält Kapillarendilatation mit Hämorrhagien. In seinem schwersten Falle, bei einer 50jährigen Dame, bestand leichte Albuminurie und sehr hoher Blutdrnck.

4. Pityriasis rosea mit einigen ungewöhnlichen Erscheinungen.

Die Besonderheiten des Falles, bei 12jähr. Mädchen, waren 1. die seltene Lokalisation des primären Herdes im Gesicht, an der linken Wange; erst 3 Tage später traten andere Plaques sehr zahlreich am ganzen Körper gleichzeitig auf; 2. auch die Hand- und Fußrücken waren stark befallen; 3. zu beiden Seiten der Spannen und an beiden FuBsohlen ziemlich viel Bläschen von Erbsengröße zwischen typischen Pit. ros. Plaques eingestreut. 
Diskussion: Adamson hat ebenfalls bei Kindern an den Händen mehrmals Bläschen bei Pityr. ros. gesehen; sie sind, da mikroskopische Bläschen in Schnitten von Plaques des Rumpfes gefunden wurden, nur als deren Exagerationen zu betrachten, nicht als konkomitierende Dyshidrosis. Pringle hat die primäre Plaque zweimal im Gesicht und auch einmal am Penis (4 Tage vor Erscheinen anderer Plaques, die erst die Diagnose klar machten), sowie gleichfalls Fälle mit Bläschenbildung gesehen, allerdings wohl nicht an Händen und Füßen.

5. Sklerema neonatoram bei einem Kinde.

Affektion begann am zweiten Lebenstage, Kind jetzt 8 Wochen alt; die charakteristische Induration teils in ausgedehnten "Platten" am ganzen Rücken, Schultern, Nacken und Oberarmen, teils in einzelnen kleinen Herden auf den Wangen und Nates vorhanden, aber indurierte diffuse Schwellungen finden sich auch an anderen Stellen. Die Haut des ganzen Körpers ist bläulichrot, die Temperatur erhöht. Die Behandlung besteht im wesentlichen darin, das Kind warm zu halten. Die Indurationen sind weicher geworden, die Prognose aber ist nicht gut.

Sibley. 1. Xanthoma multiplex?

Bei 16jähr. Jungen, der ständig fiebert, allgemeine Drüsenschwellungen, starken Husten und im Urin Albumen hat, über den ganzen Körper verbreitet ein seit 8 Jahren bestehender Ausschlag von ovalen oder runden Papeln, die rosa bis rot verfärbt sind und zum Teil gelbrötlich wächsern aussehen. Nur wenige Stellen, wie Sternum und Interskapulargegend, sind frei von diesem Ausschlage, am stärksten befallen ist die Nackengegend, wo die Haut im ganzen verdickt ist; anch an den Armen, die ebenfalls mit Papeln bedeckt sind, und am Penis und Skrotum, an Fubrücken und Sohlen starke Infiltration der Hant. Histologischer Befund der Papeln: Im Korium bindegewebige Neubildung, hier und da gelbliche Pigmentkörner und große Zellen, die eine definitive Membran und einen Inhalt von Fettgranula mit mehreren Kernen erkennen lassen; die Blutgefäße sind dilatiert.

Diskussion: Pringle, Adamson und Stowers wünschen, daß3 der Fall zur Aufklärung der Diagnose der Pathologischen Sektion überwiesen werde, Whitfield vermutet chronische inflammatorische Urtikaria.

2. Lupus vulgaris.

Sibley hat langbestehenden Schleimhaut-Lupus in Mund, Pharynx und Nase, sowie an beiden Lippen, die allgemeine Infiltration mit Knötchen aufwiesen, mit Jod. nascens, intern und lokal appliziert, bedeutend gebessert. Die 41jährige Pat. nimmt jeden Morgen sofort nach dem Frühstück 1.2 Gramm JK in 1 EBlöffel Wasser und 4,6 und 8 Stunden später je 2 EBlöffel Chlorwasser plus 1 Eßlöffel Limonade. Lokal werden die Ulzerationen des Mundes mittels eines Zerstäubers mit einem Pulver von Jodkali und Kalk zu gleichen Teilen bepudert, und der Mund wird unmittelbar nachher mit Chlorwasser gespült, das möglichst lange im Munde gehalten wird. Die Bereitung des Chlorwassers erfolgt nach Curles Vorschrift: 1 dr. gepulvertes Chlorkali $(3.7 \mathrm{~g})$ werden in eine 24 oz. (à $2 \cdot 7 g+$ ) Flasche geschūttet, 2 drr. starke Salzsäure hinzugefügt 
und das ganze 25-30 Minuten stehen gelassen, dann allmählich immer 4 oz. Wasser unter Schütteln der Flasche zugesetzt, bis diese auf $24 \mathrm{oz}$. gefüllt ist.

Diskussion: Der Fall ist wichtig und interessant; er soll in 3 Monaten wieder vorgestellt werden.

McLeod, J. M.H. OberflächlicheUlzerationen durch Kalksteine.

38jähr. Frau, Beginn der Affektion vor 6 Jahren mit roten Flecken an Knien and Beinen. Nach einigen Monaten wurden diese Flecken, in denen etwas Hartes zu fühlen war, schmerzhaft, Kalksteine erscheinen im Zentrum, durchbrachen unter Suppuration die Haut, wurden locker und fielen schließlich aus; die Ulzerationen heilten dann mit kleiner Narbe. Zur Zeit sieht man die Affektion in den verschiedenen Stadien, an den Ellbogen und Knöcheln symmetrische Plaques mit unregelmäßigen Rändern, mit Kalksteinen und Ulzerationen im Zentrum; vorn an den Knien isolierte mit Kalkstein und Entzündung; an den Fingern die gleichen Effloreszenzen und zwar am ersten Finger der linken Hand Entzündung und sklerodermieartiges Aussehen mit Kalkstein nahe der Spitze, am zweiten der Stein unter dem Nagel. Die Füße zeigten keine merkliche Affektion, nur erschien die rechte große Zehe gichtisch geschwollen. Die Kalksteine waren entweder erbsengroß und dann oval oder rund, oder sie waren größer und dann irregulär und zusammengesetzt, Konglomerate. Mit den Kalksteinen, die man bei Lupus vulgaris und anderen Krankheiten als lokale Erscheinungen sieht, hat die Affektion wohl nichts zu tun; es dürfte eine allgemeine Affektion in diesem Falle vorliegen.

Diskassion: Mac Leod hat diese Affektion noch nie gesehen; mikroskopisch scheint Neubildang von fibrösem Gewebe rings um dilatierte (wahrscheinlich Lymph-) Räume zu bestehen. Weber, Parkes nimmt Ablagerung von Phosphaten und Karbonaten in einem schwammartigen Netzwerk fibrösen Gewebes an, die als "Kalkgicht" von Widholz n. a. beschrieben wurde; auch Adamson verweist auf die Publikationen deutscher und französischer Autoren über subkutane Kalkgranulome. Whitfield erwähnt, daß Briscoe einen ähnlichen Fall beobachtete, bei dem allerdings Kalkknötchen nur an der Hand $u$. zw. nach Trauma am Daumen aufgetreten sind, bis interne Darreichung von Phosphorsäure anscheinend die Eruption zum Stillstand brachte.

Corbett. Mycosis fungoides.

Ungewöhnlich an dem Falle ist das Auftreten eines Tumors am rechten freien Rande des weichen Gaumens.

Dore. 1. A kuter Lichen planus ergthematosus miliaris.

Aussehen und Aggregierung der Knötchen genau wie bei Pityriasis rubra pilaris, nur waren die Fingerrücken völlig frei von Effloreszenzen und ein charakteristisches Lichen ruber-Knötchen in der Mundschleimhaut vorhanden.

2. Makulo-erythematōse Eruption zur Diagnose.

An Nacken, Brust, Rũcken und Oberarmen bräunlichrote Flecke in unregelmäßiger Lagerung, teils in winkeligen Linien, besonders am 
Rücken, wo sie den Spaltlinien folgen, teils mit kreisf̣̈rmigen Grenzen. Corbett stellt die Diagnose auf Parapsoriasis en plaques.

Diskussion: Pernet hält den Fall für ein Vorstadium von Mycos. fungoides, während Gray, Stowers und Pring le Parapsoriasis annehmen, aber Röntgenbestrahlung vorschlagen, auf đ్̣, M. f. gut, P. nicht reagiert.

3. Subkutane Knoten zur Diagnose.

Walnußgroße Knoten, die livide Haut bedeckt, zu beiden Seiten

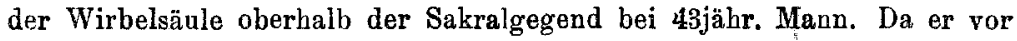
einem Jahr eine wahrscheinlich tuberkulöse Inguinaldrüsenvereiterung gehabt, dürfte es sich um ein Tuberkulid handeln, das vielleicht dem benignen Sarkoid von Boeck entspricht. (Kaposi).

Weber, Parkes. Idiopathisches multiples Pigmentsarkom

Bei 76jäbr. Mann an Extremitäten, Gesicht und Rumpf zahlreiche rote bis dunkelbraunrote Punkte und Plaques von verschiedener Form und Größe. Die branne Farbe, unter Glasdruck am besten sichtbar, rührt vielleicht von Hämosiderin ber. Die Verteilung der Effloreszenzen entspricht dem "Akro"Typ; die erhabenen Punkte und Plaques finden sich hauptsächlich an den äußersten Enden der Glieder, die Unterschenkel und Vorderarme sind stärker befallen als die oberen Teile. Eine Besonderheit bildet ein chronisches Ödem der Unterschenkel in diesem Falle. Auf der Glans penis dicht am Orificium urethrae sitzt eine hypertrophische Papel, die jetzt blaß ist, früher aber rot gewesen sein soll, gleich den im Gesicht, Nates und Abdomen vorhandenen. Einige von den größeren Plaques sind blasser und zum Teil, besonders in Zentralpartien, weniger erhaben, als ob hier eine Rückbildung stattfinde, während der Krankheitsproze $B$ in den Randpartien noch aktiv ist. Jucken besteht nicht oder nur in geringem Grade.

Diskusaion: Pringle bezeichnet den Fall als typisch für die genannte Kaposische Krankheit. Röntgenstrahlen scheinen bisweilen günstig gewirkt zu haben, doch kommen ja auch Spontanheilungen hervor, z. B. in dem ersten Falle, den er von dieser Affektion gesehen; denn diese sogenannten Sarkome sind keine Sarkome.

\section{Sitzung vom 15. Oktober 1914.}

Little, Graham. Chronische septische Papillome.

Zahlreiche feigwarzenartige weiche, nicht pigmentierte Warzen auf der unteren Hälfte der rechten Brust bei einer alten Dame. Diese Wucherungen stehen ziemlich dicht auf einer umschriebenen Area von dreieckiger Form, die vor 6 Jahren aus einem kongenitalen Naevus entstanden sein soll; sie ulzerieren nie, bluten nicht exzessiv, und einzelne verschwinden spontan, durchans normale Haut hinterlasseud.

Silbey demonstriert einen Fall, der im Februarheft des Brit. Journ. of Dermatol. publiziert wird, von lymphatischer Leukämie.

In der Diskussion weist Mc Donagh darauf hin, daß die Hauteruption auf den Oberschenkeln konfluent ist, nicht diskret wie am Rumpf 
und weniger papulös. Nach seinen Beobachtungen speziell an Juden aus dem Osten folge diesen leukämischen Eruptionen an den Oberschenkeln später eine beträchtliche Anschwellung der Inguinal- und gann eine mäBigere Vergrößerung der anderen Drüsen des Körpers. W e b e r glaubt, daß Pat. bei weiterer Entwicklung der Effloreszenzen im Gesicht eine Facies leonina oder Pseudoleprosa bekommen werde, die er selbst bei Leukämie gesehen und die Kaposi bei den irrtümlich - es waren wohl Mycosis fungoides-Fälle im prämykotischen Stadium - als Lymphodermia perniciosa beschriebenen Fällen beobachtet hat.

Pringle. Licheneruption zur Diagnose.

Bei 57jähr. Frau brüskes Auftreten intensivster Dermatitis im Gesicht, dann schnelle Ausbreitung über Kopfhaut in geringerem Grade, weiter aber mit heftiger Rötung und Schwellungen über den Körper, an den Gliedern bis zu Händen und Füßen. Fast gleichzeitig trat ein Hautausschlag auf, der persistierte, auch als die Reizerscheinungen zurückgingen. Er bestand aus zahllosen, unregelmäBig (einzeln, in Gruppen oder in größeren Flächen) verteilten Knötchen beider Formen, akuminierter sowohl wie planer. El' entstand überall, außer auf den Fingerrücken und Schleimhäuten, veranderte sich aber in der Weise, daß akuminierte die Kuppe abstießen und flach wurden, die planen aber stärkere Schuppong bekamen, die bei größeren Gruppen ein an PsorịasisPlaques erinnerndes Aussehen bewirkte. An den Beinen sah man papierdünne Desquamation, an den Palmae manum und Fußsohlen derbe Infiltration mit Rhagaden. Pringle stellt keine bestimmte Diagnose; viele Gründe sprechen für Pityriasis rubra pilaris, aber die planen Knötchen lassen es möglich erscheinen, daß Kombination mit Lichen planus vorliegt, der gleichfalls durch eine (allerdings auch noch unbekannte) toxische Ursache ausgelöst wird.

In der Diskussion erklären Adamson und Mo Leod den Fall für Pityriasis rubra pilaris, und nun schließt sich Pringle dieser Diagnose an, erinnernd an die Mitteilung, daß er Umwandlung von spitzen in flache Knötchen beobachtet hat.

Samuel. Xeroderma pigmentosum (abnorme Form)? oder Dermatit. solare?

Die Atrophie mit Teleangiektasien und Pigmentierungen am Kinn eines Mannes wird in der Disknssion verschieden beurteilt, von $\mathrm{Ad} \mathrm{a} \mathrm{m} \mathrm{s} \mathrm{on,}$ Mc Leon und Pringle aber für die Folge einer vom Pat. verheimlichten Röntgenbestrahlung erklärt.

Dore. Multiple weiche Fibrome.

Wahrscheinlich bandelt es sich um Neurofibrome.

Adamson. 1. Subkutane fibröse Knötchen im Gesicht und an den Händen.

Symmetrisch rings um die Orbitae und zu beiden Seiten der Nase mehrere wie knōcherne Prominenzen, und an einigen Fingern wie Gicht: knoten erscheinende subkutane Knoten. Mikroskopisch: Dichtes fibröses Gewebe mit geringer Infiltration von Lymphozyten an den Blutgefäßen des Koriums. Tropenkrankheit Grundou? 
2. Lupus exuberans mit miliarem Lupus, an den linken Nates, mit mehreren Attacken von "Erythem".

Pernet. Kulturen von Trichophyton sulfureum aus einer Tinea circinata am Handgelenk eines Kindes.

Mac Cormae. Lichen spinulosus mit Atrophie; (?) Tuberkulide.

An der linken Stirnhälfte eines jungen Mannes eine markstückgroße und dicht daneben sowie auf dem Hinterkopf zwei kleinere Plaques mit atrophischem Zentrum; schwärzliche hornige Auflagerungen waren vorbanden, versehwanden aber unter Lokalbehandlung mit Ac. salicyl. An anderen Körperteilen, besonders auf den linken Nates, Herde von Keratosis follicularis, aber ohne Atrophie.

In der Diskussion wird die Ansicht des Vortragenden, daB die Stirnplaques wie Lup. erythematos, aussehen, und seine Meinung bez. der Diagnose bestätigt, auch die Beziehnng des L. e. zur Tuberkulose erörtert.

Gilbs, Charles. Keratodermia blennorrhagica.

$34 j a ̈ h r$. Mann gibt an, daB er vor 5 Monaten suspekten Verkehr gehabt; 1 Monat später traten schmerzhafte Gelenkschwellungen auf (an beiden Knöcheln, Knien, Hüfte und rechtem Handgelenk) und 2 Monate post coitum AusfuB. Vor 4 Wochen bemerkte er am AuBenrand des rechten Fußes, näher an der Ferse als an den Zehen, ein kleines Bläs. chen, das hart war und beim Aufschaben keine Flüssigkeit entleerte. Weder juckte noch schmerzte es, vergrößerte sich aber. Diesem Bläschen folgte bald ein Hautausschlag. Am meisten befallen sind die Unterflächen der Füße; an den Zehen dicht bei einander zahlreiche harte, feste Knoten auf dunkelrot verfärbtem Grunde, auf den Fußrücken spärlicher, auf dem rechten 10, links aber nur auf der großen, vierten und fünften Zehe. Eine dieser Effloreszenzen des rechten Fubrückens war weich, und aus ihr ließ sich durch Punktion und Ausquetschung eine fettige Masse entleeren. Der Rest der Fulsohlen war mit glänzenden weißen Schuppen bedeckt. Auf beiden Beinen zahlreiche Eftloreszenzen, besonders an den Tibien. Aber während diejenigen an den Füljen harte Knoten aus horniger, konzentrisch angeordneter Substanz zum Teil mit schmalem hyperämischen Hofe und von dunkelbrauner Farbe waren, sind die an den Beinen befindlichen kleiner, weicher, auf Druck verschwindende Papeln, die mit trockenen Schuppen bedeckt sind. Einzelne Efforeszenzen noch an den Pubes und am Penis, denen an den Beinen gleichend, nur weiter auseinander, und an der rechten Hand drei, die denen am Fuße ähneln. Im Ausfluß Gonokokken.

Diskussion: Little, Graham hat drei analoge Fälle gesehen, von denen speziell einer dem hier demonstrierten ähnelte durch das warzenartige Aussehen einzelner Effloreszenzen. Diese befanden sich gerade auf den Ellbogen, Knien und Händen, so daß Psoriasis vorgetäuscht werden konnte. In allen drei Fällen war ebenfalls Arthritis vorhanden, und der Ausfluß bei dem Ausbruch der Hauteruption verschwanden; Heilung durch Vakzine. Pringle bezweifelt nicht die Diagnose trotz der nngewöhnlichen Lokalisation an der Hand; auch er hat drei 
Fälle mit identischen Fußsohlenerscheinungen, sowie ebenfalls mit Arthritis and Toxaemia blennorrhagica gesehen.

(Referiert nach dem Originalbericht in The Brit. Journ. of Derm 1914. Nr. 8, p. 313-335, Nr. 9. p. 353-370 und Nr. 11. p. 416-434.)

F. M ü n h h e i mer (Wiesbaden).

\section{Manchester Dermatological Society.}

Sitzung vom 3. Juli 1914.

Savatard demonstriert:

1. und 2. zwei Fälle von Lup. erythematos., der vom Nasen. rücken sich über das Gesicht, Kopf hant, Vorderarme und Finger ausbreitete bei tuberkulösem Mädchen und bei einer Frau aus tuberkulöser Familie;

3. bei 70jähriger Frau cremefarbene 'Tumoren von Stecknadelkopfgröße hauptsächlich auf den Augenlidern, deren histologische Untersuchung embryonale Basalzellen der Epidermis nachwies; ein Tumor des Vorderarms exulzerierle und hatte das Aussehen von Ulc. rodens;

4. Lupus vulgaris (sclerosus erythematoides, Leloir): ein butterartiger Fleck im Gesicht und mehrere dunkelblaurot verfärbte Stellen auf den Armen; auf Druck verschwand die Verfärbung zum Teil, diaskopisch sind miliare Tuberkel auf dem Nasenrücken nachzuweisen und subkutane Knötchen sind zu fühlen, nicht zu sehen. Keine Tuberkulinreaktion;

5. Parakeratosis variegata. Effloreszenzen von bläulichroter Farbe mit feinkleiiger Schuppung über der Area, die frischesten von ihnen papulös, Lichen planus-Knötchen ähnelnd, sind inselartig eingesprengt in die normale Haut der Vorderarm-Beugeseiten und, von da ringsherum gehend, der Handrücken und Interdigitalfächen;

6. Pagets Disease ausgehend von der linken Brustwarze.

Lancashire zeigt:

1. Erythema multiforme nach septischer Infektion der Hand, während Lymphangoitis des 1. Vorderarms an Armen, Brust und, bandförmig, am Nacken aufgetreten bei 31jährigem Manne;

2. weitverbreiteten Lichen planus bei 9jährigem Knaben;

3. Pityriasis rosea (in der Diskussion von verschiedenen Seiten für seborrhoische Dermatitis erklärt) bei einem Kinde.

Dyson demonstriert :

1. Pemphigus vegetans bei 38jähr. Mann; bald nach Erscheinen pustulös werdende Effloreszenzen, die dann papillomatösen Charakter bekommen und mit übelriechenden Belägen versehen sind an den verschiedensten Stellen des Körpers, auch an den Mundwinkeln. Von abgeheilten Effloreszenzen resistieren dunkelpigmentierte Areas. In den Belägen 